\title{
Unalternative semantics*
}

\author{
Daniel Büring \\ University of Vienna
}

\begin{abstract}
Unalternative semantics presents a new way of deriving focus alternatives from metrically annotated syntactic phrase markers, without the mediation of [F]markers or other diacritics. Using three simple rules, focus alternatives of the familiar type are derived, while certain problems such as over-focusing are automatically avoided. The formalism also allows for a new division of labor between focusing and givenness, which can be used to analyze convertible examples. Finally, applications to non-prosodic focus marking are explored.
\end{abstract}

Keywords: focus, alternative semantics, over-focusing, unalternative semantics, F-marking

\section{Introduction}

This paper explores a new way of calculating focus alternatives, as well as new ways of relating these alternatives to context and interpretation by way of felicity conditions. Work on this started out as an attempt to see if and how a standard Roothtype of alternative semantics could be implemented without the use of syntactic F-markers or similar features. The present paper, however, goes beyond that: while clearly Roothean in spirit, it generates slightly different sets of focus alternatives, which, it is argued, lend themselves more naturally to the formulation of focus interpretation rules, i.e., rules that govern, in particular, what focussings can be used in what kinds of contexts, and to what pragmatic or semantic effect.

Evidently, any set of rules that are to generate focus-related semantic information without F-markers must have direct access to those parts of intonation that are relevant for it, by assumption, metrical structure and stress; that is, the structure interpreted by these rules cannot be a pure Logical Form in the sense of most Minimalist Theories and their predecessors. It either must be a tuple of representations, at least one of them related to prosody, or a single representation including all relevant aspects, the route chosen here, mostly for reasons of simplicity: we will interpret phrase markers annotated with metrical weights, which can be mapped onto possible stress and accent patterns in the familiar ways.

Equally evidently, a system without F-markers will not structurally disambiguate F-ambiguities; rather, one structure will have to receive either multiple focus values

* Thanks to the audience at SALT 25 for their comments and suggestions. 
Unalternative semantics

(i.e., sets of focus alternatives), or a characterization of focus values that somehow subsumes all the different focus values associated with its possible foci.

\subsection{Weak restriction}

To get into the right frame of mind, consider a system in which every node in a tree with neutral stress is associated with the full set of its alternatives.

As is standardly assumed, a sentence (or a proposition) can be the FOCAL TARGET of a later sentence $S$ if and only if its meaning (or the proposition itself) is among the focus alternatives of $\mathrm{S}$ (and the same, mutatis mutandis, for all other categories). If, as contemplated just above, the focus value of a sentence with neutral intonation were to be the set of all propositions, this would mean that a structure with neutral intonation could match any focal target.

At first, this may seem acceptable. After all, a transitive sentence, for example, with neutral intonation may be interpreted as object focus, VP focus, or sentential focus. However, it cannot be interpreted as verb focus or subject focus, so the set of all propositions would be too lenient a characterization of the potential focal targets. We must begin to exclude focus alternatives. Assume the following rule:

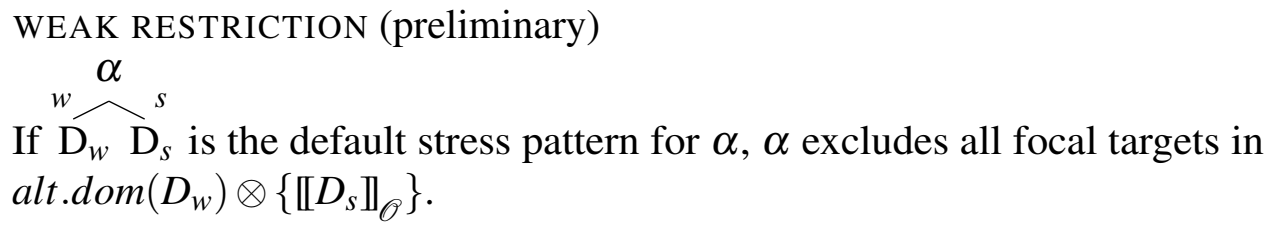

Here $\left[\mathrm{D}_{\mathrm{s}} \rrbracket_{\mathscr{O}}\right.$, as usual, is the ordinary interpretation of $D_{s}$, the metrically strong daughter, and alt.dom $\left(D_{w}\right)$ is the set of alternatives in the domain of the weak daughter $D_{w}$. $\otimes$ is a shorthand for an operation that can combine two sets of meanings (i.e., sets of focus alternatives) by point-wise combination; for example, if the sets contain one-place functions (A) and individuals (B), respectively, which are standardly combined by function application, $A \otimes B$ abbreviates $\{a(b) \mid a \in A \& b \in$ $B\}$.

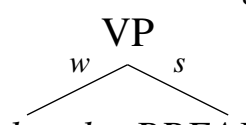

As an illustration, take a transitive VP like ordered BREAKfast, with default stress on the right sister, as $\alpha$. By rule (1), it excludes as focal targets all properties composable by applying a possible alternative to 'ordered' to the concept 'breakfast' . By assumptions, possible alternatives to 'ordered' are simply all relations expressible by transitive verbs ${ }^{1}$ therefore, ordered BREAKfast excludes any focal targets of the

1 We cannot use the whole set of relations between individuals, because that would include functions like the one mapping 'breakfast' to the property 'paid for lunch', leading us to wrongly exclude 'paid for lunch' as an alternative to ordered breakfast. This is a version of a general complication that 
form 'relation applied to breakfast', basically: any property having to do with breakfast. Conversely, it allows as focal targets all VP meanings (meanings in $\operatorname{dom}(V P) \subseteq D_{e t}$, to be precise) minus those in alt.dom $\left(V_{t r}\right) \otimes\left\{\llbracket\right.$ breakfast $\left.\rrbracket_{\mathscr{O}}\right\}$; this includes 'ordered lunch', 'ordered drinks' etc., as well as 'left', 'cried', 'ate dinner' etc., i.e., object focus alternatives and almost all VP focus alternatives. Excluded are alternatives that would be in the standard alternative set of ORDERED breakfast.

It will be good to have a way to characterize such restrictions on focal targets using formulae. The restriction of ordered BREAKfast just discussed, for example, will be written as in (2).

\section{$R$ breakfast}

read: exclude any property derivable by applying a relational (transitive verb) meaning to the concept 'breakfast'

As just discussed, (2) is short for a set of meanings- here: properties - which we will call the UNALTERNATIVES of VP (since they are the ones that cannot be focal targets). I write $\llbracket \psi \rrbracket_{\mathscr{U}}$ for the set of $\psi$ 's unalternatives. To make clearer the relation to standard alternative semantics, note the following correspondence:

$$
\operatorname{dom}(\psi) \backslash \llbracket \psi \rrbracket_{\mathscr{U}} \approx \llbracket \psi \rrbracket_{\mathscr{F}}
$$

In other words, we get the set of possible focal targets for a constituent $\psi$ as the complement of the unalternatives relative to the domain of $\psi$. The resulting set is almost, but usually not exactly, the same as $\llbracket \psi \rrbracket_{\mathscr{F}}$ in standard Roothean Alternative Semantics. For example [[ordered BREAKfast $\left.]_{\mathrm{F}}\right]_{\mathscr{F}}$ is the set of all properties, i.e., $\operatorname{dom}(V P)$, whereas, as just discussed, $\operatorname{dom}(V P) \backslash\left[\right.$ ordered BREAKfast $\rrbracket_{\mathscr{U}}$ equals $\operatorname{dom}(V P)$ minus the 'related in some other way to breakfast' properties. We will return to the consequence of this difference in Sections 2 and 3; this, at any rate, is why I used ' $\approx$ ' ('has the same function in the overall system'), rather than '=' in (3).

At every branching node $\alpha$, rule (1) effectively filters out all alternatives that rely on alternatives to the weak daughter, $D_{w}$, alone. Note that this is different from saying that only the strong daughter, $D_{s}$, may introduce alternatives; we still permit lots of $\alpha$-alternatives that are not among $\llbracket D_{w} \rrbracket_{\mathscr{O}} \otimes$ alt $\operatorname{dom}\left(D_{s}\right)$ (VP meanings like 'left', 'ate dinner,...' in the case of (2)), just not those that happen to be in

arises in alternative semantics, as pointed out in Bonomi \& Casalegno 1993 (see Büring in press: sec 5.4.1 for discussion); all that is required in the system proposed in the present paper is some kind of restriction to 'natural meanings'. For example, restricting the set of possible transitive verb alternatives to the set of functions from pairs of individuals to events in which the first participates as an agent and the second as a theme or patient (essentially the solution pursued in Bonomi \& Casalegno 1993) would do as well. 


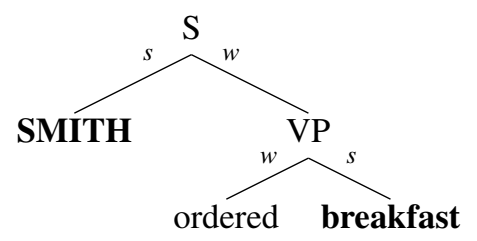

Figure 1: Narrow subject focus involves a non-default strong-weak pattern at the $S$ level, but still default stress within VP.

alt.dom $\left(D_{w}\right) \otimes\left\{\llbracket D_{s} \rrbracket_{\mathscr{O}}\right\}$ ('ate breakfast, 'skipped breakfast',...). This seems an empirically adequate characterization of the VP's potential focal targets.

We actually need to make one immediate refinement to this: a branching node with normal stress pattern may be part of the focus, or part of the background, depending on what happens higher up. Consider for example the tree in figure 1, which corresponds to a sentence with narrow subject focus, with a single pitch accent on Smith (signalled by capitals). Yet, even though there are no pitch accents in VP, there is still the default stress pattern according to which breakfast is stronger than ordered (signaled by boldface). That is to say, the same stress pattern that occurs in a default broad focus (or narrow object focus) VP can also appear if the same VP is entirely within the background.

Since the structure in Figure 1 should allow for alternatives like 'Jones ordered breakfast', 'Franklin ordered breakfast' etc., we should never, at the lower (VP) level, exclude the literal meaning of a neutral node. This is formalized in rule (4).

WEAK RESTRICTION

$\alpha$

If $\mathrm{D}_{w} \mathrm{D}_{s}$ is the default stress pattern for $\alpha, \alpha$ excludes all focal targets in $\left(\right.$ alt.dom $\left.\left.\left(D_{w}\right) \backslash\left\{\llbracket D_{w} \rrbracket_{\mathscr{O}}\right\}\right) \otimes\left\{\llbracket D_{s} \rrbracket_{\mathscr{O}}\right\}\right)$

Applying rule (4) to the VP in the tree in figure 1, we now allow the set of all VP meanings, except those that are relations to breakfast other than ordering breakfast. The unalternatives are now written as in (5).

$R^{\backslash \text { ordered }}$ breakfast

read: exclude any VP meaning composed of a relation other than 'ordered' applied to 'breakfast'

Rule (4) applies at the S level as well, provided that — unlike in the tree in figure 1VP is metrically strong and the subject metrically weak (i.e., the default). The result 
for Smith ordered BREAKfast is (6) (see also the tree in figure 2(ii)).

$$
x^{\backslash \text { Smith }} \text { ordered breakfast }
$$

read: exclude any proposition composed of the property 'ordered breakfast' to an individual other than Smith

This allows for propositions like 'it started to rain', 'Franklin arrived' (i.e., sentential focus) as well as 'Smith left', 'Smith bought a paper' (VP focus), as well as 'Smith ordered lunch' (object focus); it excludes 'Jones ordered breakfast', 'Hyam order breakfast' etc., i.e., subject focus.

\subsection{Propagation}

Unfortunately, the restriction expressed in (6) above also allows for 'Smith ate breakfast', that is, it ignores the restriction introduced at the VP level, (5) on the previous page. To remedy this, we need to make sure that lower restrictions get propagated to higher nodes. The rule for this is given in (7).

\section{PROPAGATION}

any branching node $[\alpha \mathrm{D} 1 \mathrm{D} 2]$ excludes all focal targets in $($ alt.dom $(D 1) \otimes$ $\left.\llbracket D 2 \rrbracket_{\mathscr{U}}\right)$ as well as those in $\left(\llbracket D 1 \rrbracket_{\mathscr{U}} \otimes\right.$ alt.dom $\left.(D 2)\right)$

Rule (7) takes (6) and turns it into (8) in the configuration Smith ordered BREAKfast (i.e., default weak-strong).

$$
x R^{\backslash \text { ordered }} \text { breakfast }
$$

read: exclude any proposition composable from a relation other than 'ordered' applied to a (random) individual and 'breakfast'

While (8) allows 'Jones ordered breakfast', (8) and (6) combined rule it out. In fact their combination can be written as a single restriction, $Q^{\text {smith ordered }}$ breakfast. The whole derivation is summarized in Figure 2.

Note that propagation runs vacuous at the VP level. This is because terminals do not, by definition, introduce any restrictions. For the same reason, only the first clause of rule (7) leads to unalternatives in (8) $\left(\llbracket\right.$ Smith $\rrbracket_{\mathscr{U}}=\emptyset$, and hence $\llbracket$ Smith $\rrbracket_{\mathscr{U}}$ $\otimes$ alt.dom (ordered breakfast $)=\emptyset$; a case in which both clauses of rule (7) are active is discussed around Figure 5(i)).

Suppose for simplicity that the only alternatives in the domains of 'Smith', 'order' and 'breakfast' are 'Jones', 'paid for' and 'lunch', respectively. Then $Q \backslash$ smithordered breakfast predicts the suitability of focal targets in the way summarized in the table 

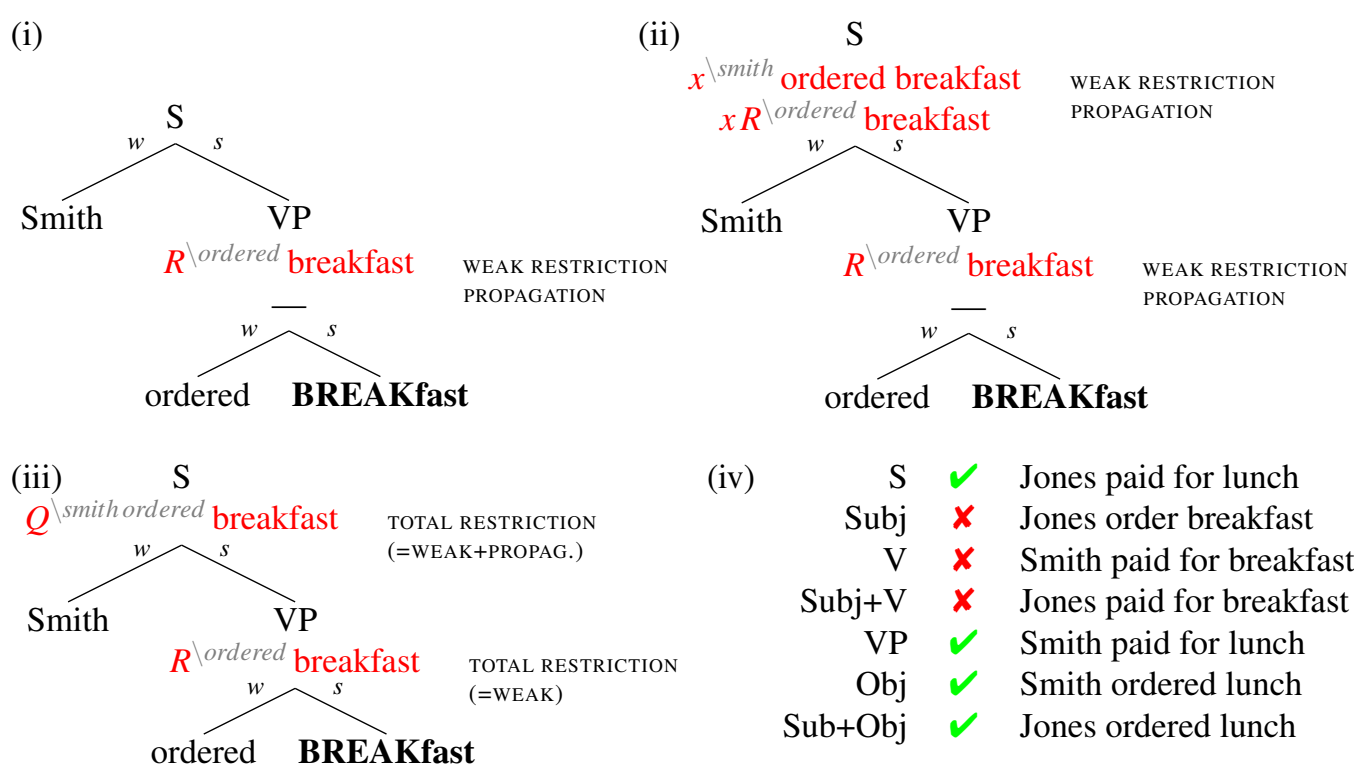
(iv) $\mathrm{S} \vee$ Jones paid for lunch
Subj $x$ Jones order breakfast
$\mathrm{V} \times$ Smith paid for breakfast
Subj+V $\quad \boldsymbol{X}$ Jones paid for breakfast
VP $\checkmark$ Smith paid for lunch
Obj $\checkmark$ Smith ordered lunch
Sub+Obj $\checkmark$ Jones ordered lunch

Figure 2: Unalternatives calculated at the VP, (i), and S level, (ii). (iii) shows how the (weak) restriction introduced at the $\mathrm{S}$ level, and the weak restriction propagated from the VP level can be combined into a single restriction. The resulting predictions regarding what can and cannot be a focal target according to $Q^{\backslash \text { smithordered }}$ breakfast are shown in the table in (iv). The leftmost column indicates what kind of focus this would correspond to in a system using F-marking.

in Figure 2(iv). These are exactly the restrictions we want.

\subsection{Strong restriction}

Now let us turn to cases of non-default intonation. In keeping with the overall metrical tree framework we are using, such cases involve PROSODIC REVERSAL, i.e., the switching of weak and strong among sister nodes. In these cases, a stronger restriction is introduced: The strong sister must be (part of) a focus, with the weak sister in the background.

STRONG RESTRICTION

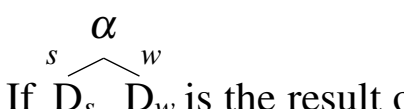
If $\mathrm{D}_{s} \mathrm{D}_{w}$ is the result of prosodic reversal, $\alpha$ excludes all focal targets in alt.dom $(\alpha) \backslash\left(\left(\right.\right.$ alt .dom $\left.\left.\left(D_{s}\right) \backslash \llbracket D_{s} \rrbracket_{\mathscr{O}}\right) \otimes\left\{\llbracket D_{w} \rrbracket_{\mathscr{O}}\right\}\right)$

For example, ORDERED breakfast would exclude all VP meanings except those 
(i)

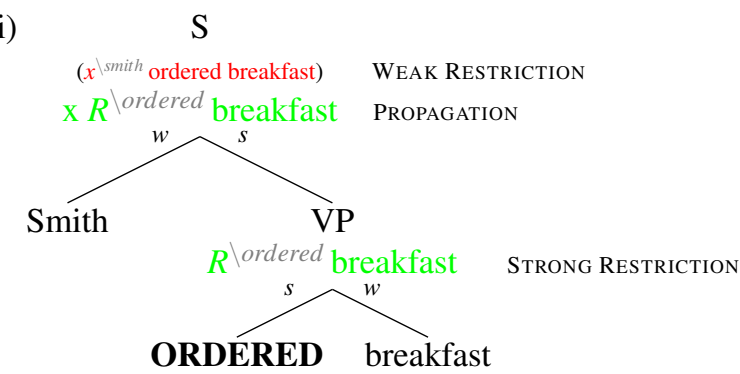

(ii)

$x^{\text {smith }}$ ordered breakfast

(x $R^{\text {lordered }}$ breakfast) PROPAGATION

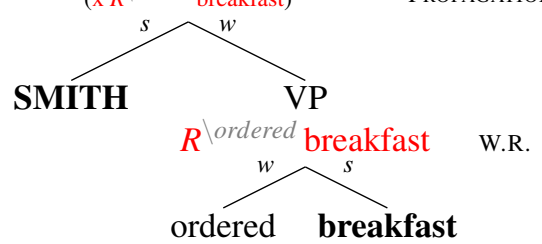

Figure 3: Strong restriction, applied at the VP- and S-levels (3(i)/3(ii), respectively); note that in the latter case, as in the tree in figure 1, normal metrical weights, $w-s$, are assigned within the VP.

composed of a relation other than 'ordered' applied to 'breakfast'. Using the conventions for writing restrictions as formulae employed so far, this could be written as in (10a). An equivalent but more readable notation is given in (10b), where a green formula characterizes the focal targets that are allowed according to rule (9).
a. $Q^{\backslash R^{\backslash \text { ordered }} \text { breakfast }}$
b. $R$ \ordered breakfast

Both ways of writing restrictions are equivalent, but the first is more suitable for writing weak restrictions (the few things that are excluded as focal targets), while the second is more suitable for strong restrictions (the few things allowed to be focal targets). I will therefore use them both in what follows, urging the reader to keep in mind that the formal system only knows one kind of restriction, the unalternatives.

Full derivations are given in the trees in figures 3(i) and 3(ii); similarly to Figure 2, the restrictions introduced by Propagation and Restriction can be subsumed by a single restriction, which in this case is the same as the result of Strong Restriction (plus Propagation where applicable) alone; in such cases, the redundant restriction is parenthesized and in a small font. ${ }^{2}$ The predictions this makes are summarized in the table in Figure 4.

Finally, what if we apply prosodic reversal twice? This is unproblematic if the lower reversed node is within the promoted constituent at the higher reversed node. The result, shown in Figure 5(i), is a regular narrow focus. Prosodic reversal within a demoted constituent, on the other hand, will always yield inconsistent restrictions at the higher reversal site, as in Figure 5(ii): one restriction wants the focal target to be

2 Likewise, a strong restriction subsumes what would be the result of Weak Restriction appliedaccording to weak/strong labels - to the prosodically inverted sisters; so we do not, in principle, need to restrict rule (4) above to sisters with default metrical weights. 
Unalternative semantics

\begin{tabular}{|c|c|c|c|c|}
\hline \multicolumn{3}{|c|}{$\begin{array}{c}\text { Smith ORDERED breakfast } \\
\times R^{\backslash \text { lordered breakfast }}\end{array}$} & \multicolumn{2}{|c|}{$\begin{array}{c}\text { SMITH ordered breakfast } \\
x \backslash \text { smith } \text { ordered breakfast }\end{array}$} \\
\hline $\mathrm{S}$ & $x$ & Jones paid for lunch & $\mathrm{S}$ & $x$ \\
\hline Subj & $x$ & Jones order breakfast & Subj & $\checkmark$ \\
\hline $\mathrm{V}$ & $\checkmark$ & Smith paid for breakfast & V & $x$ \\
\hline Subj+V & $\checkmark$ & Jones paid for breakfast & Subj+V & $x$ \\
\hline VP & $x$ & Smith paid for lunch & VP & $x$ \\
\hline Obj & $x$ & Smith ordered lunch & Obj & $x$ \\
\hline $\mathrm{Sub}+\mathrm{Obj}$ & $x$ & Jones ordered lunch & $\mathrm{Sub}+\mathrm{Obj}$ & $x$ \\
\hline
\end{tabular}

Figure 4: Predictions for prosodic reversal at the VP and S level, respectively.

(i)

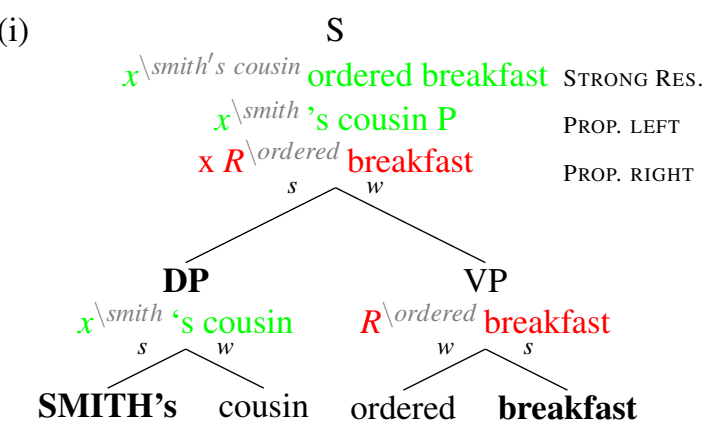

(ii)
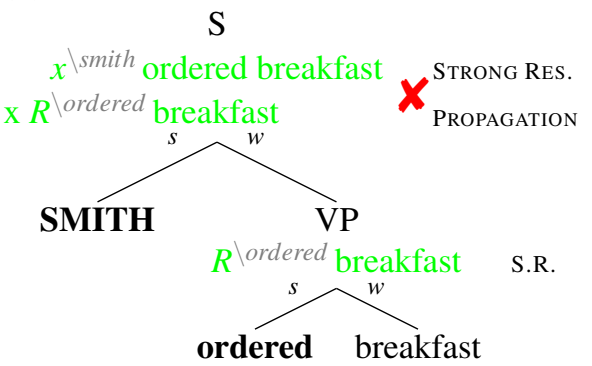

Figure 5: 5(i) shows prosodic reversal within a prosodically promoted constituent; the

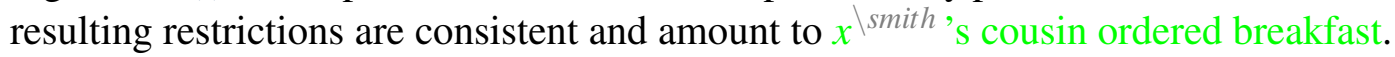
5(ii), prosodic reversal within a weak sister, leads to inconsistent restrictions.

about someone ordering breakfast, while the other wants the focal target to be about someone in some relation other than ordering to breakfast. This means that between them, these restrictions exclude any proposition as a focal target. I will assume that this is impossible, though more will be said in Section 4.

\subsection{Focus retrieval}

In order to have a precise way of feeding focus alternatives into the pragmatics or semantics, I will add a mechanism of FOCUS RETRIEVAL, closely modelled on Rooth's (1992) squiggle operator in its individual case.

(11) FocUs RETRIEVAL (see (13) and (22) below for possible amendments)

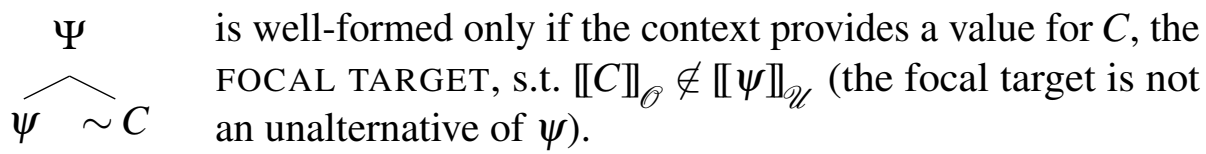


(i)

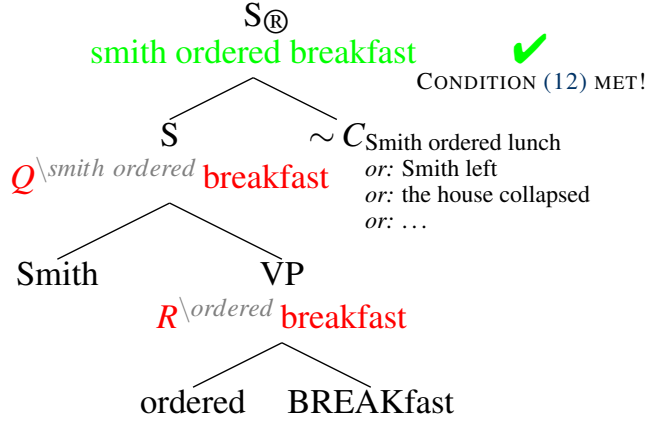

(ii) $x^{\backslash \text { smith }}$ ordered breakfast $x$ ordered breakfast CONDITION (12) MET!

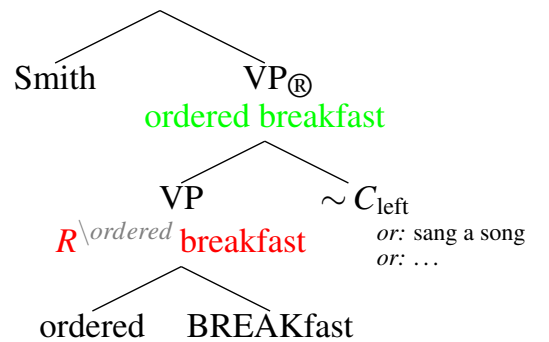

Figure 6: Retrieval resets the unalternatives, thereby meeting condition (12). Note that the result of resetting, plus propagation and weak restriction where applicable, automatically leads to unalternatives which only allow the literal meaning, as required by condition (12).

If so, $\llbracket \Psi \rrbracket_{\mathscr{U}}$ may (but need not be) set to alt.dom $(\Psi) \backslash\left\{\llbracket \Psi \rrbracket_{\mathscr{O}}\right\}$ (i.e., $\Psi$ ).

In short, if the value of $C$ is not excluded by the restrictions accumulated on $\psi$, the

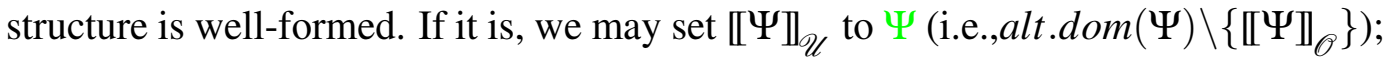
this amounts to saying that $\Psi$ cannot target any alternative any more. $\Psi$ is now bound to function as background, and any focussing within it is henceforth inaccessible. ${ }^{3}$ Nodes where this option, which I will call FOCUS RESET, is chosen will be marked by a subscripted $₫$ (for 'retrieval') in the trees that follow.

Finally, based on rule (11) on the previous page, condition (12) on root nodes can be imposed to make sure that any focus in a sentence will indeed be retrieved, i.e., find a pragmatic focal target.

$$
\begin{aligned}
& \text { CONDITION ON } \llbracket]_{\mathscr{U}}: \text { 'Use Focus!' } \\
& \text { For every root node } \mathbf{R}, \llbracket \mathbf{R} \rrbracket_{\mathscr{U}} \text { must be } \operatorname{alt} . \operatorname{dom}(\mathbf{R}) \backslash\left\{\llbracket \mathbf{R} \rrbracket_{\mathscr{O}}\right\} \text { (i.e., } R \text { ). }
\end{aligned}
$$

Condition (12) can only be met if focus is retrieved at least once in the tree; after the focus is retrieved, the option of setting $\left[\Psi \Psi \mathbb{U}_{\mathscr{U}}\right.$ to $\Psi$ is tantamount to "starting with a blank slate'. As long as only Weak Restriction applies, (11) and (12) are rather easy to satisfy, in particular since propagating the result of Focus Reset always yields unalternatives that satisfy condition (12). This is demonstrated in Figure 6, especially the tree in figure 6(ii).

3 This is the, optional, counter-part of Rooth's (1992) clause that says that $\llbracket \Psi \rrbracket_{\mathscr{F}}=\emptyset$. 
Unalternative semantics

This concludes our first run-through of the system proposed here. I will now consider a number of applications beyond standard contrastive focus.

\section{Answer focus}

Rule (11) above captures the standard case of contrastive focus, i.e., cases where the focal target is e.g., a single proposition or property. But what about focussing in answers to (constituent) questions, in which case the focal target appears to be a question denotation, say, a set of propositions? This case is not entirely straightforward in the system proposed here, for the following reason: Take Smith ordered BREAKfast again, the unalternatives of which are characterized by $Q^{\backslash \text { smith ordered }}$ breakfast. This correctly excludes focal targets like 'Smith paid for breakfast'.

But what if it answers the question What did Smith do?, which it intuitively can? According to standard question semantics, the denotation of that question does contain, among others, the proposition 'Smith paid for breakfast', which, as we just saw, is an unalternative. This state of affairs will obtain generally: Some of the propositions in the denotation of a constituent question will be among the unalternatives to the intuitively correctly focussed answer. We cannot, therefore, require that all elements in the question denotation need to be compatible with the restrictions of its answer (which would be the straightforward analogon to the set case in Rooth 1992).

The simplest way to deal with this is a weaker condition on answer focus: that at least some element in the question denotation needs to be consistent with the restrictions of the answer. This is in fact easily done by minimally amending rule (11) above as in (13).

$$
\begin{aligned}
& \text { AMENDMENT TO RULE }(11) \\
& {[\psi \sim C] \text { is well-formed only if the context provides a focal target to antecede }} \\
& C \text { s.t. } \llbracket C \rrbracket_{\mathscr{O}} \notin \llbracket \psi \rrbracket_{\mathscr{U}} \text { or } \llbracket \mathbf{C} \rrbracket_{\mathscr{O}} \nsubseteq \llbracket \psi \rrbracket_{\mathscr{U}} \text { (whichever one is defined),... }
\end{aligned}
$$

(13) still correctly rules out answers in which an entirely wrong constituent is focussed. Take SMITH ordered breakfast as an answer to What did Smith do? The answer only tolerates focal targets of the form $x^{\text {smith }}$ ordered breakfast; the question meaning can be characterized as 'smith $Q$ '. These two are disjoint, failing the putative condition on answer focus in (13).

What (13) does not rule out are cases in which only a sub-constituent of the intuitively correct focus is focussed, e.g., Smith ORdered breakfast as an answer to the same question. This answer requires focal targets characterized by

4 An earlier version of this paper had a major error in this definition; thanks to Nadirah Porter-Kasbati and Clemens Mayer for setting me straight on this. 
Smith $R$ \ordered breakfast; and in fact all those propositions are in the denotation of What did Smith do?, meeting (13), the weaker condition on answer focus we are contemplating.

Rather than looking for a version of the answer condition that would rule out the overly narrow focus, I propose to rule out this case by an additional condition on prosodic demotion.

\section{CONDITION ON PROSODIC DEMOTION}

A prosodically demoted constituent has to be given (i.e., its meaning must be contextually salient)

This implies that Smith ORdered breakfast is in fact a felicitous answer to What did Smith do?, but only in a context in which breakfast is given, i.e., the concept 'breakfast' has been made contextually salient before, e.g., by previous mentioning. This predicts the pattern generally assumed to be correct, e.g., in Selkirk 1984, 1995, Rochemont 1986, or Schwarzschild 1999 (see Büring 2006: for detailed discussion of such cases and their implications). A different possible route to capturing answer focus will be sketched in Section 5 below.

\section{Overfocussing}

A pleasant consequence of restricting focus alternatives the way we do here is that we immediately exclude what is often referred to as OVERFOCUSSING. In standard alternative semantics it always holds that $\llbracket S_{\alpha F} \rrbracket_{\mathscr{F}} \subseteq \llbracket S_{\beta F} \rrbracket_{\mathscr{F}}$, where $\alpha$ is a sub-constituent of $\beta$ and $\mathrm{S}_{\alpha F}$ and $\mathrm{S}_{\beta \mathrm{F}}$ are identical phrase markers, except that $\alpha$ (and only $\alpha$ ) is F-marked in $\mathrm{S}_{\alpha F}$, and $\beta$ (and only $\beta$ ) is F-marked in $\mathrm{S}_{\beta \mathrm{F}}$. Given that the focus constraint always requires something to be an element or a subset of $\llbracket \alpha \rrbracket_{\mathscr{F}}$, it follows that whenever a narrow focus on some constituent $\alpha$ is contextually permitted, broader focus on any $\beta$ that dominates $\alpha$ is permitted, too. In some cases, such as (15), this leads to wrong predictions.

$$
\begin{aligned}
& \text { a. (What did Smith do to Jones?-) \# Smith [kissed JONES }]_{\mathrm{F}} \\
& \text { b. (Smith bit Jones.— No, \# Smith [kissed JONES }]_{\mathrm{F}}
\end{aligned}
$$

Main stress on an object is compatible with F-marking on VP. And the focus alternatives to a F-marked VP include, among many others, all properties of the form ' $R$ Jones', i.e., all properties in answers to the question in (15).

The only type of solution to this problem that I am aware of invokes transderivational constraints, such as AvOIDF (Schwarzschild 1999). In the present system, on the other hand, we do not have the automatic superset relation mentioned 
Unalternative semantics

\begin{tabular}{cc} 
WEAK & STRONG \\
\hline functional & lexical \\
head & complement \\
left projection & right projection
\end{tabular}

Figure 7: Some structural defaults, in descending order of importance.

above. Sloppily speaking, F-alternatives to a VP focus sentence do not include the F-alternatives to a transitive $\mathrm{V}$ focus sentence. Generally, a larger constituent does not have those focus alternatives which would rely only on its weak daughter: $\llbracket$ kissed JONES $\rrbracket_{\mathscr{U}}$ does not permit alternatives like 'hugged Jones', 'hit Jones' etc. Therefore, VP focus as in (15) is incompatible with any focus target that fixates on John. The following lemma holds:

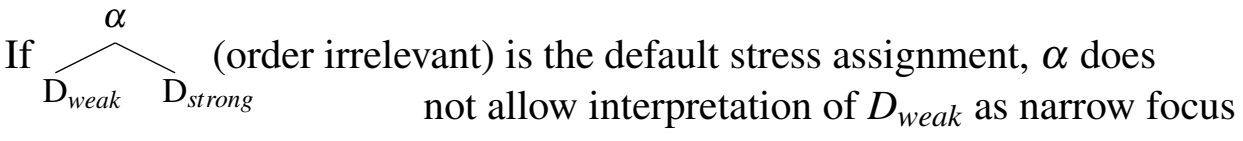

On the face of it this only excludes a subset of overfocussing cases. What about the case where narrow focus on a strong daughter is wrongly realized by F-marking the mother? Such a case is illustrated in (17).

$$
\text { Who did Smith kiss? - Smith [kissed JONES }]_{\mathrm{F}}
$$

The answer is that on the present perspective, there is nothing wrong with this example. Without F-markers, focus on the mother and focus on the strong daughter are the same thing. The only instance in which overfocussing leads to testably false predictions is if it involves prosodic reversal, or, more precisely, the lack thereof. So really, all we need to avoid is 'underbackgrounding', and this is indeed a built-in property of the Unalternatives system.

It is worthwhile to mention at this point that prosodic reversal is not necessarily the same as deaccenting. To appreciate this point, it is necessary to look at other kinds of prosodic defaults than left=weak, right=strong. The table in Figure 7 gives a list of other prosodic defaults, which do not necessarily coincide with linear order.

In OV languages like Dutch and German, the effect of the head-complement relation on stress pattern is particularly obvious. A sentence final VP has the main stress on the object, rather than the final verb; see Figure 8(i).

Since this is the default stress pattern, the focus (un)alternatives in this structure are the same as in the neutral English case. Focussing the verb narrowly, however, does not primarily result in deaccenting the DP (it will still bear a secondary, prenuclear accent), but an additional accent on the verb. Yet the effect on the focus alternatives is the same as with object-deaccenting in English: the prosodically 
(i)

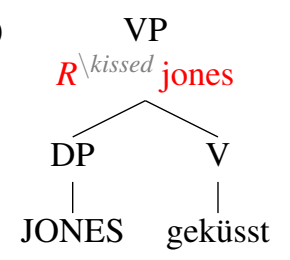

(ii)

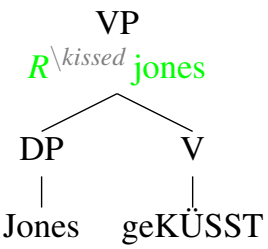

(iii)

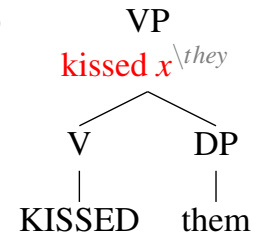

(iv)

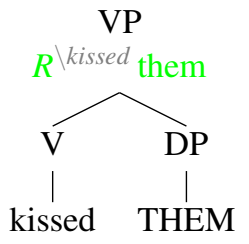

Figure 8: Left-headed defaults: Default (i) and reversed (ii) complement-head pattern in the German VP 'kissed Jones', and with a functional element as complement—default (iii), and reversed (iv).

promoted daughter — geküsst — must be (part of) a focus, and the demoted daughter must not be; see Figure 8(ii).

A similar effect can be observed in head-initial phrases with a functional complement: the tree in figure 8(iii) shows the default stress assignment, while the tree in figure 8(iv) is unambiguously narrow object focus, even though it need not involve deaccenting (again, kissed may bear pre-nuclear stress and accent), but obligatory re-accenting.

\section{Second occurrence focus}

Since Weak and Strong Restriction are based on metrical weights (rather than pitch accents), they apply to cases of Second Occurrence Focus as well. An example is given in (18), where the associated SOF skimmed is marked by stress (prosodic reversal), but no pitch accent.

(The kids only skimmed the book.) Even JOHN only skimmed the book.

A full analysis of (18) is given in Figure 9. The focus sensitive elements are treated as in Rooth 1992 as restricted by a contextual variable, which in turn is (obligatorily) restricted by a minimally c-commanded focus variable, with the squiggle operator interpreted according to rule (11) above.

Note that, in accordance with that rule, the set of unalternative is reset (®) by the squiggle at VP and S. In both instances, this is crucial: at the S-level because of condition (12) above, the requirement that there be no unused restrictions at the root level; and at the VP level, because otherwise we would get a set of restrictions which cannot be met. To see that, contrast the tree in figure 9 with that in Figure 10, where the VP unalternatives are propagated up to $S$, instead of being reset: between the propagated (un)alternatives $x \cap R \backslash$ skimmed the book and the newly introduced strong restriction $x \backslash^{j o h n}$ only skimmed the book, there is simply no proposition that could 
Unalternative semantics

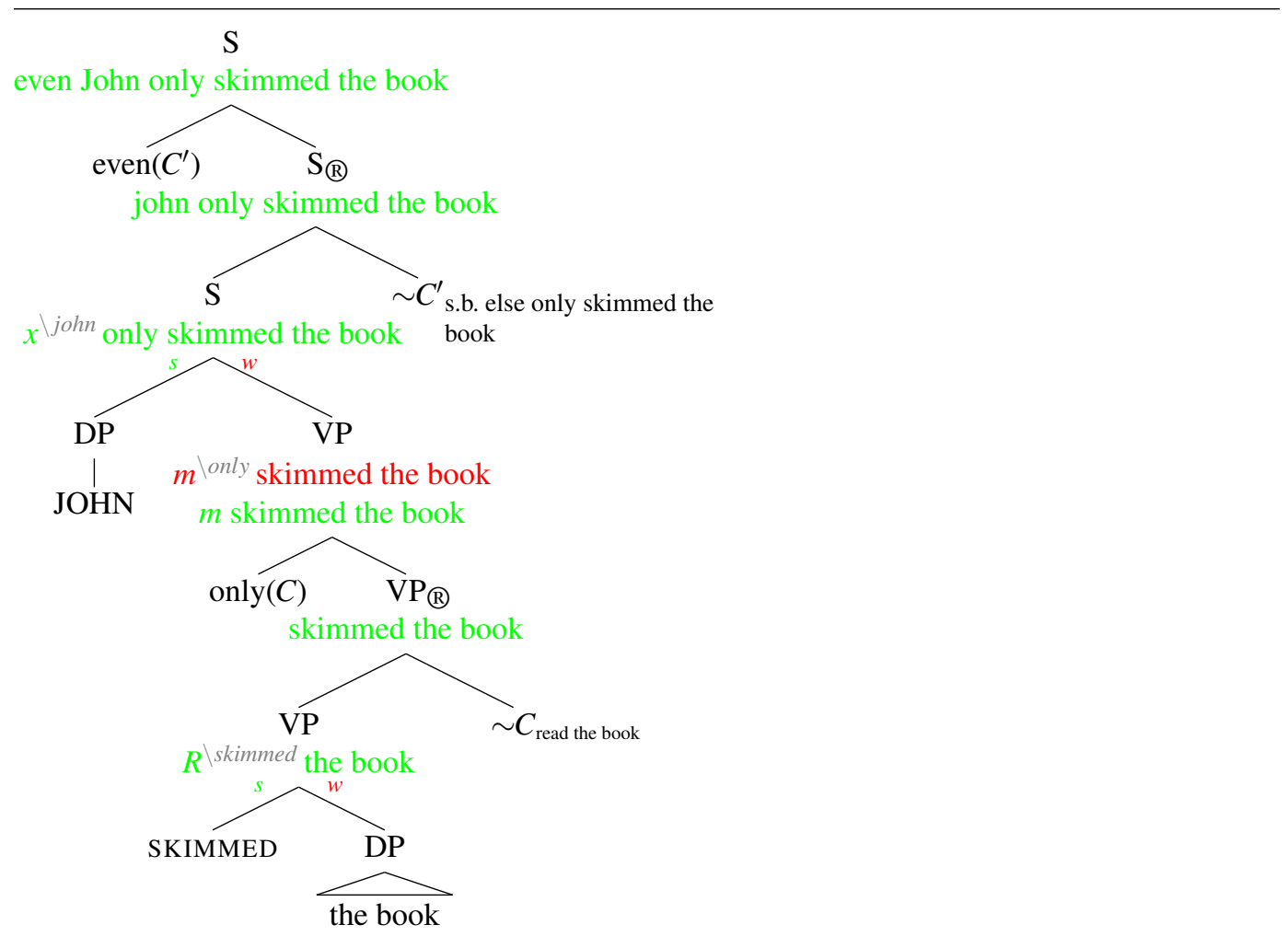

Figure 9: Second Occurrence Focus

serve as a focal target and satisfy both restrictions.

As said in Section 1.3 above, I take this configuration to be excluded. In fact, this now follows: no focal target can be found to retrieve the focus in Figure 10, consequently the unalternatives at the root level do not accord to condition (12) above.

While this problem can be circumvented as in Figure 9, by resetting the unalternatives after retrieving the lower focus, this is not possible if the lower focus cannot be retrieved before the next prosodically reversed node is reached. This corresponds exactly to the 'no crossing focal dependencies' restriction I argued for in Büring 2013/15, illustrated in Schwarzschild's (2006) example (19).

(What did John only eat in PARIS?)
a. \#John only ate CRÊPES in Paris.
b. \#John only ate crêpes in PARIS.

Here, Paris should be the focus of only, while crêpes wants to be an answer focus, and therefore be retrieved by a sentence-level squiggle. These two dependencies- 


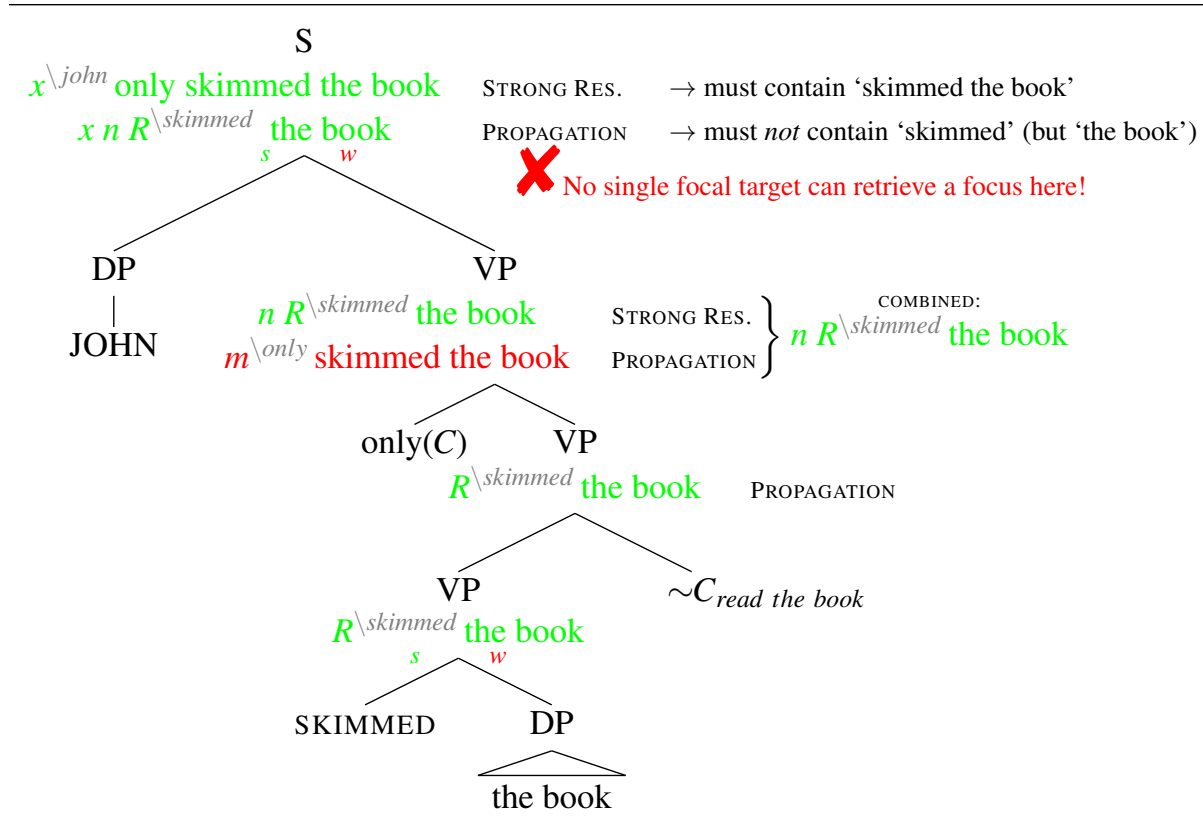

Figure 10: Conflicting restrictions as a result of not resetting focus within a prosodically demoted constituent (compare to Figure 9).

unlike those between only and its focus skimmed, and even and its focus John in Figure 9-overlap, which renders the sentence ineffable in this context.

According to the analysis in Büring 2013/15, crossing dependencies are impossible due to an F-realization condition. In the present setup, they automatically result in conflicting restrictions: we have a case of prosodic reversal within a prosodically demoted constituent (see Section 1.3 above), without the possibility of intermediate retrieval (unlike in the tree in figure 9). Three ultimately ill-formed derivations are shown in Figures 11 and 12.

(19a), the intuitively correct answer focussing, is impossible since only wants to associate with Paris, which is blocked by the unalternatives, see Figure 11.

For (19b) there are two possible derivations, both of which fail: If we reset the unalternatives after (successfully) retrieving the focus that associates with only, as in the tree in figure 12(i), no contrasting focal target at the S-level is permitted at all (since there was no prosodic reversal after resetting). If we do not reset the unalternative, as in the tree in figure 12(ii), we likewise fail at the S-level because the focal target is not allowed to involve 'Paris'. 


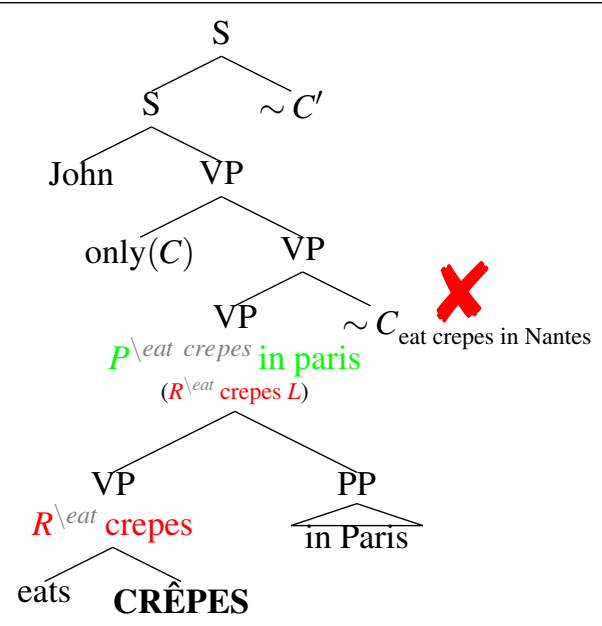

Figure 11: Stress on crêpes/VP makes it impossible to have 'eat crêpes in L' as a focal target.

\section{Excursus: givenness, contrast, and question under discussion}

Rule (11) above, with or without the amendment in (13), does not require that the focal target itself, i.e., $\llbracket C \mathbb{\rrbracket}_{\mathscr{O}}$, be contextually salient (given). The condition on prosodic demotion, (14), on the other hand, does requires givenness, but only of the background of the focus, not of the target of the entire focus domain, and only if it was prosodically demoted. ${ }^{5}$

This mix, I will now argue, is exactly what is needed. To see why, consider Wagner's $(2006 ; 2012)$ by now famous convertible examples in (20).

(20) Mary's uncle, who produces high-end convertibles, is coming to her wedding. I wonder what he brought as a present.

a. He brought a [CHEAP convertible].

b. \#He brought a [RED convertible]

c. He brought a red CONVERTIBLE.

The focal target for (20a) should be 'he brought a high-end convertible'. But that proposition is not given in the context. What is given is convertible, the element demoted by prosodic reversal. All of this accords to rules (11) and (14), but would be prohibited if the focal target itself needed to be given.

Now, the reader might object, couldn't the contrast in example (20a) be merely

5 Thus, for example, ordered in the answer in What did Smith order? - Smith ordered BREAKfast, need not, by (14), be given, since no prosodic demotion has taken place; see Büring forthcoming, sec.3, for why that might be a Good Thing. 


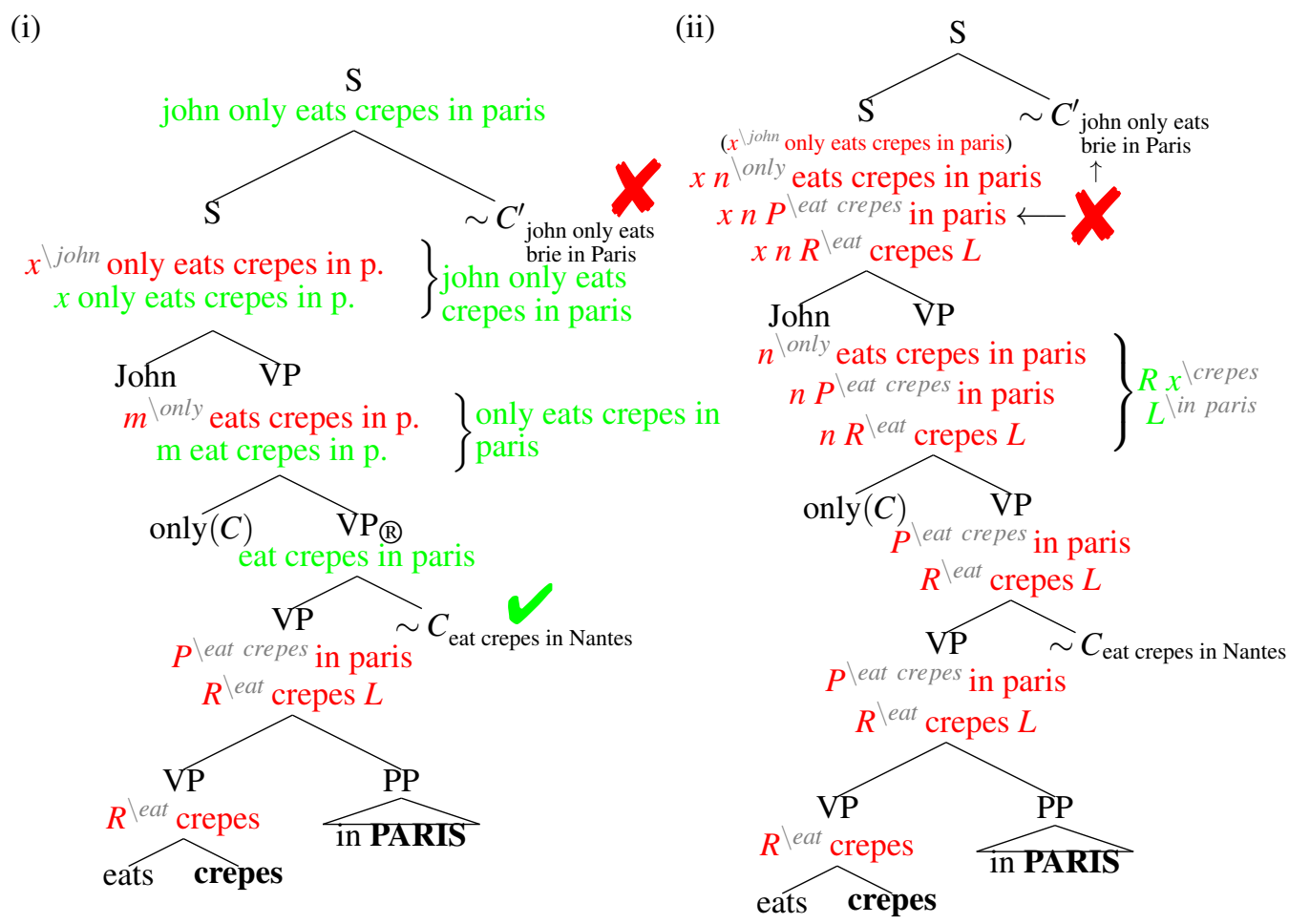

Figure 12: Whether we reset the focus or not: crêpes cannot be focussed across only... Paris.

'cheap convertible'-v.-'high-end convertible', in which case the focal target-'highend convertible' - would be given? Not if we want to-as Wagner 2006, 2012 urges us to-exclude RED convertible in variant (20b) on the grounds that it fails to contrast properly with 'high-end convertible'. To decide that, it is necessary to look at the larger constituent, a point forcefully made by Katzir's (2013) example (21).

(21) The people in this club are very particular about the cars they collect. Mary, for example, collects high-end convertibles.

a. And John collects CHEAP convertibles.

b. And John collects RED convertibles.

While 'he brought a red convertible, he didn't bring a high-end convertible' is indeed a non-contrast, 'he collects red convertibles, he does not collect high-end convertibles' is just fine, which intuitively accounts for why deaccenting convertible in the context of red is odd in (20b), but fully natural in (21a) (see Katzir 2013 for a formalization of this intuition). But that means that the pertinent condition on what does and what does not count as contrasting cannot just compare red convertible 
and its putative focal target 'high-end convertible', but rather has to look at the entire sentence (or at least the VP) (he) brought a/collects red/cheap convertible(s) vis-à-vis its focal target 'he brought a/collects high-end convertible(s)'; and that focal target is not contextually given even in the well-formed example (20a) above. Therefore, as stated at the outset, we must not demand that the focal target in its entirety be given.

Does this mean that any proposition whatsoever can function as the focal target, as long as it properly contrasts? Probably not, because in that case nothing would stop us from choosing e.g., 'he brought a blue convertible' as the focal target in (20b), which-being a proper contrast to red convertible both intuitively and in the formal sense of innocent exclusion proposed in Katzir 2013-would wrongly predict (20b) to be acceptable. And while the question whether Mary got a red or a blue convertible for her wedding might not seem to be particularly relevant in the context set up in (20), I would hesitate to blame the infelicity of (20b) on that alone: to the extent that it can be justified to mention that the convertible is red, it should be equally relevant if it were blue. Put simply, as far as the relevance of the question 'red or blue' is concerned, example (20b) above should probably be no odder than (20c), leaving the actual contrast unaccounted for.

I would like to propose instead that the focal target must be uniquely identifiable, i.e., it must be clear to the hearer which value the speaker had intended for it. Given that the focal target is the value of the focus variable $C$, which in turn is nothing but an unpronounced pronoun, this seems a rather plausible requirement. In (21) on the preceding page (the case of convertible collectors), this requirement is clearly met, given that the contrasting alternative 'collect high-end convertibles' has just been mentioned, i.e., that we are dealing with a contrastive focus.

In (20) (the wedding gift), on the other hand, any one of the question alternatives is equally (non-)salient, which is to say, the hearer has no way of knowing whether the intended focal target is 'blue convertible', 'yellow convertible', 'green convertible' etc. The only uniquely identifiable contrasting alternative is 'he brought a non-red convertible', i.e., the disjunction of all innocently excludable focus alternatives. And that, I submit, is not a relevant alternative.

The intuition behind this is, once again, quite clearly brought out by the paraphrase 'he brought a red convertible, as opposed to a non-red convertible', which is odd in this context. I will speculate a little bit more on how to formalize this in a moment. For now, the following will do as a stand-in:

Alternative Amendment to Rule (11) ABOVE $[\psi \sim C]$ is well-formed only if the context provides a value for $C$, the FOCAL TARGET, s.t. 
a. $\quad \llbracket C \rrbracket_{\mathscr{O}} \notin \llbracket \psi \rrbracket_{\mathscr{U}}$ (the focal target is not an unalternative of $\psi$ ) (=rule (11) above), and

b. if the focal target were true (and the ordinary meaning false), rather than the other way around, this would make a difference to the participants, and

(RELEVANT CONTRAST)

c. the value for $C$ is uniquely identifiable for the participants (general condition on pronouns)

It is important to stress that condition (22) on the preceding page must replace (13), rather than supplement it. If a question could still generally license answer focussing as long as some question alternative is not excluded, this would still license the deaccenting in RED convertible in (20b) above, on the grounds that e.g., the question alternative 'he brought a blue convertible' is not an unalternative (this parallels Wagner's $(2006 ; 2012)$ point that simple givenness within a larger focus does not suffice to license deaccenting).

But if condition (22) on the preceding page (or its eventual formalization) is a requirement on all foci, we must make sure that we do not rule out answer focus in general. Take a run-of-the-mill case like (23).

(Who did Jones meet? - ) Jones met SMITH.

By parity of reasoning, none of the individual question alternatives is more salient than the others, so-absent a more specific context - the hearer is left with 'Jones met someone other than Smith' as the only identifiable focal target. But note now that 'Jones met Smith, as opposed to someone else' does already sound a lot better than 'Mary's uncle brought a red convertible, as opposed to a non-red one'. Put differently, the question 'did Jones meet Smith or someone else?' is one that the questioner in example (23) will very likely be interested in (it is, for example, a proper subquestion to the question actually asked); it is, on the other hand, a stretch to claim that the question 'did Mary's uncle bring a red or a non-red convertible' should be of any interest in (20).

If I had a full semantic analysis of expressions like $p$ as opposed to $q$ or $p$ rather than $q$, I could now formalize RELEVANT CONTRAST (in the sense of condition (22b)) as "whatever is required for "[ordinary meaning] as opposed to [focal target]" to be felicitous', perhaps something along the lines of 'whether $p$ and not $q$ or $q$ and not $p$ would make a difference to the participants'. Absent that, I offer these phrases as a diagnostic for the presence of a RELEVANT CONTRAST, leaving the formalization for a future occasion.

Pursuing this line of analysis amounts to saying that there is no true answer-toconstituent-question focus; focus always marks a contrast between exactly two 
meanings, i.e., contrastive focus. This idea has predecessors in Schwarzschild's (1999) treatment of answer focus using the existential closure of the question as a GIVENness antecedent; but crucially, the focal target on the view proposed here is subject to a much stronger condition than GIVENness - so as to rule out example (20b) above — and it is not required to be contextually salient—so as to rule in (20c). There are also affinities to the suggestion made in Wagner 2012, p.sec.1.2.6 that any one of the propositions in the denotation of a constituent question is salient enough to be, in present terms, the contrastive focal target for the answer; but crucially, on the present view, individual question alternatives are not made salient by a constituent question (for example, they cannot license deaccenting ${ }^{6}$ ), nor is contrastiveness of a single question alternative sufficient for contrastive focussing in the answer —otherwise, example (20b) above would be ruled right back in. ${ }^{7}$ I believe that an analysis along the lines sketched in this excursus would indeed be the first to correctly predict all the examples discussed in this section and the papers referenced in it.

Summing up, in this excursus I argued for the condition on focussing, rule (11) above, amended as in (22), which is both stronger and weaker than the usual conditions on focussing (such as Rooth 1992 or Schwarzschild 1999); stronger in that it incorporates a substantial notion of contrast (the lesson learned from Wagner 2006), and weaker in replacing 'contextual saliency (before the utterance)' by 'identifiability (after the utterance)'.

Deaccenting, or more generally: prosodic reversal, too, is subject to this condition on focussing; in addition, however, it is restricted by a plain givenness requirement, (14) above; both conditions combined are strictly stronger than any condition on anaphoric deaccenting or backgrounding in the literature.

Finally, no requirement that backgrounds in general need to be contextually given is entailed be the rules here; they only need to be if deaccented (i.e., usually post-focally).

6 That is, (i) is impossible, even though, according to Wagner 2012, p.sec.1.2.6, the question in (i) makes the proposition 'Sally arrested Smith' (among countless others, of course) 'available'.

(i) (Who arrested Smith. - - \# I REAlly DOUBT that Sally arrested Smith.

See Büring in press, p., sec.5.3.4 for a detailed version of this worry.

7 'Mary's uncle brought a blue convertible' is of course one of the question alternatives in example (20) above, so by Wagner's own reasoning it should suffice as a focal target to He brought a RED convertible. 


\section{Outlook: non-prosodic focus marking}

In this section I will sketch some applications of Unalternative Semantics to nonprosodic focus marking. Examples will be drawn from Hausa, a Chadic language spoken in Nigeria and neighboring countries.

\subsection{Focus indifference}

Hausa is an SVO language. A sentence with neutral word order, such as (24) is possible as an answer to 'What did he stab the wild animal with?', 'What did he stab with a knife?', 'What did he do?' etc., as well as to correct e.g., he stabbed his friend with a knife, he stabbed the wild animal with a spear etc. That is to say, in a neutral word order sentence, any constituent can be interpreted as focus (except the subject, to which we return in Section 6.3 above).

$$
\begin{aligned}
& \text { Yaa sòokee naamà-n dà wukaa. } \\
& \text { 3SG.PERF stab meat-DET with knife } \\
& \text { 'He stabbed the wild animal with a knife.' }
\end{aligned}
$$

Remarkably, there appear to be no prosodic or other differences between the different focus interpretations of example (24). The sentence, even when spoken, is simply neutral with respect to its focus interpretation. Transparently, this effect can be achieved by assuming that neither metrical nor other structural properties introduce unalternative of any kind in Hausa; anything can be interpreted as a focal target.

This does not mean, however, that focus is irrelevant for the grammar of Hausa, as we will see momentarily.

\subsection{Focus positions}

Hausa has a left-peripheral focus position, by which I mean that anything in that position is inevitably interpreted as focussed. Apart from the non-standard constituent order, a filled focus position can be detected by a different form of the verb, called the RELATIVE form, as well as, optionally, a focus marker nèe to the right of the constituent in focus position. ${ }^{8}$

8 Examples from Newman 2000. 
Unalternative semantics

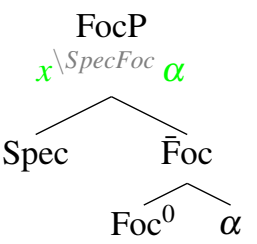

Figure 13: Modelling a focus position like the one found in Hausa.

a. Kiifii nèè Kande takèè dafààwaa.

fish FOC K. 3SG.RELATIVE.CONTINUOUS cooking.

'Kande is cooking the fish.' object focus

b. Baayan bishiyàà yakèè.

behind tree 3SG.REL.CONT

'He is behind the tree.' locative focus

A sentence like (25a) can answer the question 'What is Kande cooking', but not 'Who is cooking the fish?' or 'What is Kande doing with the fish?'; analogously for (25b). That is why the position kiifii/baayan bishiyàà occupy in (25) is called a focus position.

To model this using unalternatives we can assume that the focus position STRUCTURALLY introduces a strong restriction, as sketched in Figure 13. This requires $\alpha$ (e.g., Kande takèè dafààwaa in example (25a)) to be in the background, and SpecF to at least contain a focus, which seems to be the desired result. ${ }^{9}$

The restriction in Figure 13 does not force focus movement, which, as discussed in Section 6.1 on the preceding page is correct: all elements in the focus position are focal, but not all foci appear in the focus position.

I should say that presumably the state of affairs described in this subsection could be

9 Green \& Jaggar 2003, p.26 report that at least some speakers accept sentence with two foci, one preposed, one in situ, as in (i) (similar examples ar discussed in Hartmann \& Zimmermann 2007: sec.2.3).

(i) Kun ga mààtaataa à makaíantaa, koo?

2PL.PERF see wife.my at school Q

Aa'àà, yaarònkà nee mukà ganii à kààsuwaa.

no son.you FOC 1PL.RELATIVE.PERF see at market

'Did you see my wife at school? — No, I saw your son at the market.'

This is ruled out by Strong Exclusion as in Figure 13, which requires 'I saw at the market' to be backgrounded. To allow for it, a new variant of restriction would be needed, which says 'left daughter must be/contains a focus, right daughter may or may not', which we could write as: SpecFoc Q. 


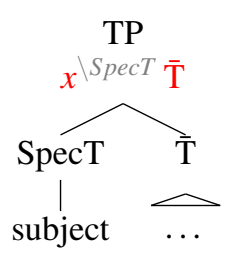

Figure 14: The unfocus nature of the subject position in Hausa.

modelled using F-markers (and some kind of checking or F-criterion) as well, though both the optionality of focus movement and the fact that the element in the focus position may merely contain the pragmatic focus ('pied piping') will presumably require non-trivial assumptions, while they follow from Strong Restriction directly.

\subsection{Unfocus positions}

I have already hinted at a final feature of focussing in Hausa in Section 6.1 above: in a sentence in which the focus position is empty, the subject cannot be interpreted as narrow focus. That is, example (24) above cannot answer the question 'Who stabbed the wild animal with a knife?' In order for a subject to be interpreted as narrow focus, it has to appear in the focus position (which, given the SVO order in Hausa is mainly detected by the relative form of the verb, as well as, optionally, the focus marker). The structural subject position in Hausa is thus an UNFOCUS POSITION.

As this nomenclature may lead the reader to suspect already, there is a straightforward implementation of this in unalternative semantics, given in Figure 14, which, as in the case of the focus position above, relies on unalternatives being introduced by non-metrical, syntactic aspects of the structure. Figure 14 says that the subject (specifier of TP) cannot be a narrow focus in Hausa. Accordingly, if it is to be narrowly focussed, it has to move to the focus position (or, at least in principle, some other position fit for the purpose, though I know of no instance of this).

This case is particularly interesting from the point of view of unalternative semantics, because it is far from clear how an unfocus position should be modelled by familiar methods. Clearly, no structural marking of any particular position as focus would achieve the desired effect. But the problem is more fundamental: Even if we availed ourselves of a feature like G(iven), B(ackground), or T(opic) this would be of no use here. Neutral word order, with the subject in canonical position, may and in fact will be used in out-of-the-blue, or whole focus, sentences. The subject need neither be given nor in the background of a focus, it just cannot itself be (or contain) the focus.

The closest one could come using features would be to introduce a feature, call 
it [-F], meaning 'I do not tolerate the focus here'. However, such a feature could not, by itself, guide the calculation of focus alternatives, for the reasons just discussed: we cannot know whether the actual focus is in, on, or dominating $\overline{\mathrm{T}}$ instead, or none of the above (in case TP is embedded in a clause with e.g., narrow subject or verb focus). The calculation of focus alternatives would still have to rely on F-markers, with $[-\mathrm{F}]$ functioning merely to block the appearance of an $\mathrm{F}$ in a position so marked. ${ }^{10}$

All this illustrates that unalternative semantics as sketched here is not just a notational variant of standard alternative semantics. It makes very particular predictions about what is a natural class of focus interpretations, i.e., what kinds of different focal targets may be available for a given prosodic and/or syntactic structure (as well as its sub-parts).

\section{Summary}

There are some obvious essential features to unalternative semantics that distinguish it from standard alternative semantics and similar approaches: It works without F-markings, it rules out overfocussing without the need for transderivational constraints, and it allows for discontinuous foci.

There are other features in the package I offered here which fit in very naturally with UAS, but may not be essential for, nor, in principle, exclusive to it: Its particular division of labor between focussing and givenness, the possibility to toy around with stronger contrast conditions, its applicability to non-prosodic focus marking.

Finally, it is worthwhile to point out some conceptual points which I find interesting about this project. It seems to me an odd feature of all frameworks for focus semantics I have seen, used, and advocated so far, that they model what, to my mind, is clearly a relational phenomenon (the pragmatic distinction between focus and background) using a privative category $\mathrm{F}$ (ocus). This is not just a terminological issue. What I mean is that there are always rules that influence the calculation of focus alternatives in response to whether that node is F-marked or not, but never in response to the relation of that node to its sister. In many cases, this seems related to the fact that the realization of focus is itself taken to be a privative matter, namely the presence versus absence of a pitch accent.

10 Even such a system would not be equivalent to the Unalternative System explored here, since it does not rule out overfocussing. If, for example, a transitive clause with default metrical weights yielded the pattern of marking on the right, which should allow for an interpretation as VP focus, I can see no way to block the introduction of alternatives like 'ate breakfast' at the VP level while still allowing e.g., 'ran home', i.e., VP alternatives not relying on object alternatives alone.

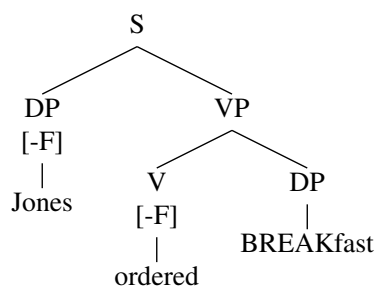


Unalternative semantics, as opposed to that, directly relates one relational concept - stress or metrical strength - to another-focus v. background, and part of its simplicity relates, I would argue, to that fact. Among other things it follows naturally that things in focus should not be expected to share any prosodic properties, nor should things in the background; things in focus may be metrically weak, and unaccented (as well as, of course, strong and accented), and things in the background may bear various degrees of stress and be accented, all of which strike me as correct and welcome predictions.

Apart from that, unalternative semantics suggests a different, hopefully more appropriate, taxonomy of relations between sister constituents: It distinguishes only two structural relations between sisters, default and reversed, where standard focus semantics distinguishes four $(\mathrm{F}+\emptyset, \mathrm{F}+\mathrm{F}, \emptyset+\emptyset, \emptyset+\mathrm{F})$. At the same time, it semantically distinguishes those two cases that come out indistinguishable in standard alternative semantics, namely $\mathrm{F}+\emptyset$ versus $\emptyset+\mathrm{F}$ (and everything else).

Many consequences of this remain to be explored, details to be filled in, changes to be made. Hopefully, I have piqued the readers' interest in following this program further, or even contributing to it.

\section{References}

Bonomi, Andrea \& Paolo Casalegno. 1993. Only: Association with focus in event semantics. Natural Language Semantics 2(1). 1-45.

Büring, Daniel. 2006. Focus projection and default prominence. In Valéria Molnár \& Susanne Winkler (eds.), The Architecture of Focus Studies in Generative Grammar 82, 321-346. Berlin, New York: Mouton De Gruyter.

Büring, Daniel. 2013/15. A theory of second occurrence focus. Language As a Cognitive Process/Language, Cognition and Neuroscience 30(1-2). 73-87. doi:10.1080/01690965.2013.835433.

Büring, Daniel. forthcoming. Focus, questions and givenness. In Klaus von Heusinger, Edgar Onea \& Malte Zimmermann (eds.), Questions in Discourse, Holland: Brill.

Büring, Daniel. in press. Intonation and Meaning Surveys in Semantics and Pragmatics. Oxford University Press.

Green, Melanie \& Philip Jaggar. 2003. Ex-situ and in-situ focus in Hausa: Syntax, semantics, and discourse. In Jacqueline Lecarme, Jean Lowenstamm \& Ur Shlonsky (eds.), Research in Afroasiatic Grammar III (Current Issues in Linguistic Theory 2), 187-213. Amsterdam: John Benjamins Publishing Company.

Hartmann, Katharina \& Malte Zimmermann. 2007. In place-out of place: Focus in Hausa. In Kerstin Schwabe \& Susanne Winkler (eds.), Information Structure and 
Unalternative semantics

the Architecture of Grammar: A Typological Perspective, 365-403. Amsterdam: John Benjamins.

Katzir, Roni. 2013. A note on contrast. Natural Language Semantics 21(4). 333-343. doi:10.1007/s11050-013-9095-8. http://dx.doi.org/10.1007/s11050-013-9095-8.

Newman, Paul. 2000. The Hausa Language. New Haven \& London: Yale University Press.

Rochemont, Michael. 1986. Focus in Generative Grammar. Amsterdam/Philadelphia: John Benjamins.

Rooth, Mats. 1992. A theory of focus interpretation. Natural Language Semantics 1. 75-116.

Schwarzschild, Roger. 1999. Givenness, AvoidF and other constraints on the placement of accent. Natural Language Semantics 7(2). 141-177.

Schwarzschild, Roger. 2006. Focus interpretations: Comments on Geurts and van der Sandt. Theoretical Linguistics 30(1). 137-147. doi:10.1515/thli.2004.003.

Selkirk, Elisabeth O. 1984. Phonology and Syntax: The Relation between Sound and Structure. Cambridge, Mass.: MIT Press.

Selkirk, Elisabeth O. 1995. Sentence prosody: Intonation, stress, and phrasing. In John A. Goldsmith (ed.), The Handbook of Phonological Theory, 550-569. London: Blackwell.

Wagner, Michael. 2006. Givenness and locality. Semantics and Linguistic Theory (SALT) 16. 295-312. doi:http://dx.doi.org/10.3765/salt.

Wagner, Michael. 2012. Focus and givenness: A unified approach. In Ivona Kučerová \& Ad Neeleman (eds.), Contrasts and Positions in Information Structure, 102147. Cambridge: Cambridge University Press.

Daniel Büring

Department of Linguistics

University of Vienna

Sensengass 3a

1090 Vienna

Austria

daniel.buring@univie.ac.at 